\title{
Frontal encephalopathy related to hyperinflammation in COVID-19
}

\author{
Ilaria Cani ${ }^{1}$ - Valentina Barone ${ }^{1} \cdot$ Roberto $^{\prime}$ Angelo $^{2}$ (D) Lara Pisani $^{3} \cdot$ Vincenzo Allegri $^{4} \cdot$ Luca Spinardi $^{5}$. \\ Paola Malpassi ${ }^{6} \cdot$ Luca Fasano $^{3} \cdot$ Rita Rinaldi $^{2} \cdot$ Stefano Fanti ${ }^{4} \cdot$ Pietro Cortelli $^{1,7} \cdot$ Maria Guarino $^{2}$
}

Received: 25 June 2020 / Revised: 3 July 2020 / Accepted: 4 July 2020 / Published online: 11 July 2020

(c) Springer-Verlag GmbH Germany, part of Springer Nature 2020

Dear Sirs,

Since coronavirus disease 2019 (COVID-19) outbreak, neurologic manifestations have been increasingly reported including encephalopathy; however, the underlying pathophysiology remains mostly unclear [1]. Neurotropism of severe acute respiratory syndrome coronavirus-2 (SARSCoV-2) has been suspected [2], though neuropathological studies did not show specific brain changes [3].

Besides the SARS-CoV-2 infection, a dysregulated immune response resulting in a massive release of proinflammatory cytokines is involved in pathogenesis of severe COVID-19 manifestations and multi-organ failure [4]. This systemic hyperinflammatory state may be involved in neurologic impairment, as well.

Ilaria Cani, Valentina Barone and Roberto D'Angelo These authors share co-first authorship.

Roberto D'Angelo

roberto.dangelo@aosp.bo.it

1 Department of Biomedical and NeuroMotor Sciences, Alma Mater Studiorum-University of Bologna, Bologna, Italy

2 IRCCS Istituto delle Scienze Neurologiche di Bologna, UOC Interaziendale Clinica Neurologica Metropolitana (NeuroMet), Neurologia S. Orsola-Malpighi Hospital, Via Albertoni, 15, Building\#2, 40138 Bologna, Italy

3 Respiratory and Critical Care Unit, St. Orsola-Malpighi University Hospital, Bologna, Italy

4 Nuclear Medicine Unit, St. Orsola-Malpighi Hospital, Bologna, Italy

5 NeuroRadiology Unit, St. Orsola-Malpighi Hospital, Bologna, Italy

6 Laboratorio Unico Metropolitano AUSL Bologna, Bologna, Italy

7 IRCCS Istituto delle Scienze Neurologiche di Bologna, Bologna, Italy
We report a case of COVID-19-related encephalopathy, questioning temporal relations between infection, cytokine storm, and neurologic involvement.

A 77-year-old female, with no history of neurological disease, presented with impaired consciousness after 18-days history of SARS-CoV-2 infection and acute respiratory distress requiring invasive mechanical ventilation (Fig. 1). Patient was placed on hydroxychloroquine, levofloxacin, and piperacillin/tazobactam.

Despite a remarkable respiratory improvement, at time of first neurologic evaluation, patient presented awake but mutacic, without any goal-directed behavior. No meningeal irritations or focal signs were found. Stimulus-induced myoclonus and positive primitive reflexes (blinking, left grasp) were observed. Electroencephalogram (EEG) recording showed a generalized slowing activity, prevalent in frontal regions. A magnetic resonance imaging (MRI) displayed diffuse white-matter lesions consistent with chronic small vessel disease without contrast enhancement (Fig. 2).

Cerebrospinal fluid (CSF) analysis detected normal white blood cell counts and mild increase of the blood-brain barrier permeability $(\mathrm{CSF}$ protein $=56 \mathrm{mg} / \mathrm{dl}$, reference range $<50 ; \mathrm{CSF} /$ serum albumin ratio $=15,6$, reference range $<7,4)$.

CSF reverse transcription-PCR (RT-PCR) for SARSCoV-2 was negative. Additional CSF studies, including oligoclonal bands, neurotropic virus, bacterial cultures, and autoimmune encephalitis antibody panel, were all negative.

Cytokines levels were tested both in CSF and blood documenting a significant increase of interleukin-6 (IL-6) (55.1 and $9.1 \mathrm{pg} / \mathrm{ml}$ respectively, reference range $<5,9)$ and interleukin-8 (IL-8) (106 and $2721 \mathrm{pg} / \mathrm{ml}$, respectively, reference range $<70$ ) (Fig. 1).

${ }^{18} \mathrm{~F}$-fluorodeoxyglucose-positron emission tomography $\left({ }^{18} \mathrm{~F}-\mathrm{FDG}-\mathrm{PET} / \mathrm{CT}\right)$ scan showed a spread frontal lobe hypometabolism (Fig. 2).

She was treated with intravenous methylprednisolone $60 \mathrm{mg}$ for 10 days. 


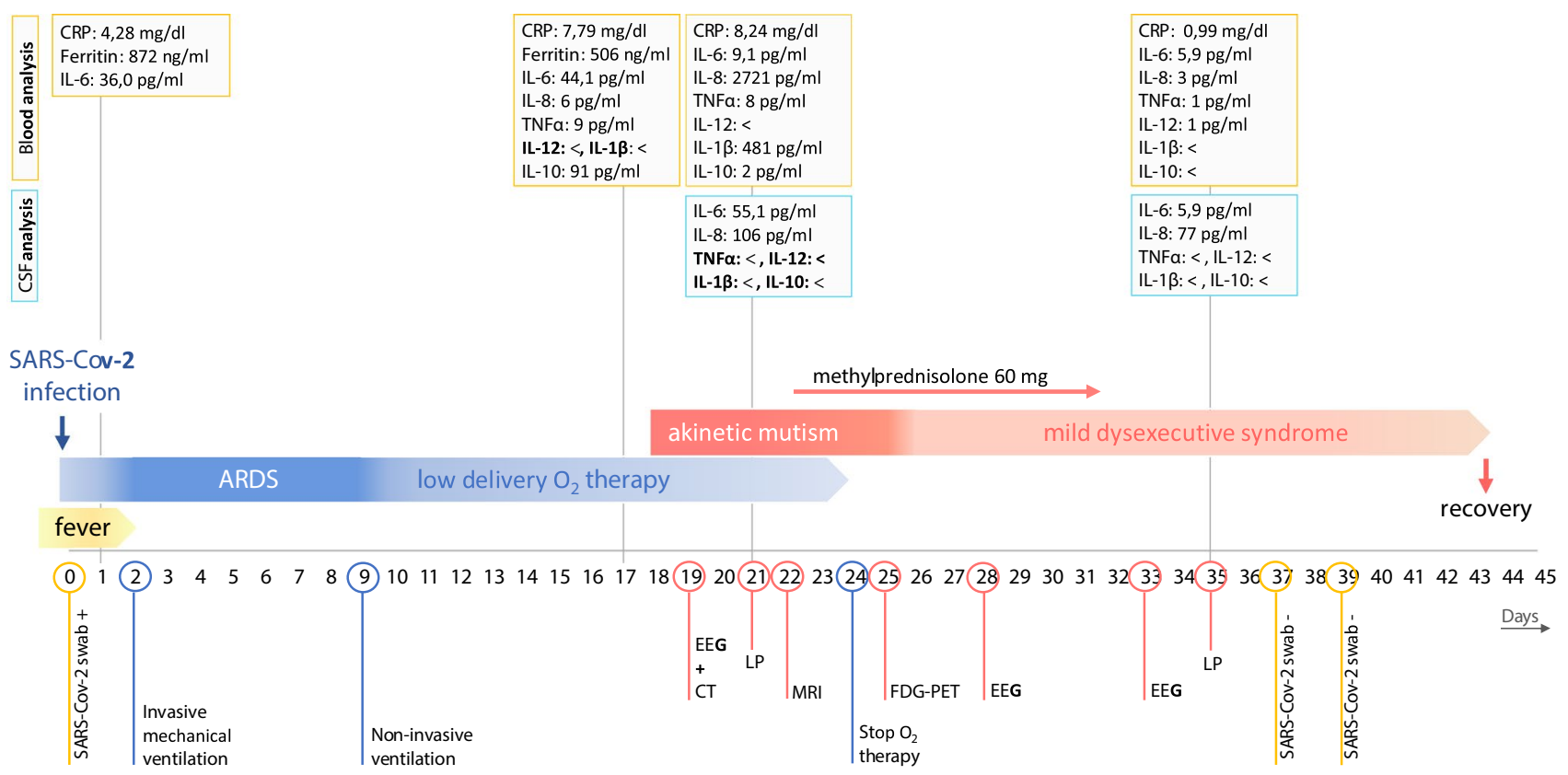

Fig. 1 Clinical symptoms, diagnostic work-up, treatment, and inflammatory profile during the disease course. CSF cerebrospinal fluid, $A R D S$ acute respiratory distress syndrome, $E E G$ electroencephalography, $C T$ computed tomography, $L P$ lumbar puncture, $M R I$ magnetic resonance imaging, FDG-PET fluorodeoxyglucose-positron emission tomography, $C R P$ C-reactive protein, $I L-6$ interleukin-6, $I L-8$ interleukin-8, TNF $\alpha$ tumor necrosis factor-alpha, $I L-12$ interleukin-12, $I L-1 \beta$ interleukin-1Beta, $I L-10$ interleukin-10;<, under limit of detection. Reference range: $\mathrm{CRP}<0,50 \mathrm{mg} / \mathrm{dl}$; ferritin $11-306 \mathrm{ng} / \mathrm{ml}$; IL- $6<5,9$ pg/ml; IL- $8<70$ pg/ml; TNF $\alpha<8,1$ pg/ml, IL- $10<9$ pg/ml
Over time, patient slowly but progressively improved until complete recovery of speech and environment interaction. Serial EEGs showed gradual reduction of previous slow activity. The second lumbar puncture detected a mild increase of blood-brain barrier permeability (CSF protein $=66 \mathrm{mg} / \mathrm{dl}$, reference range $<50 ; \mathrm{CSF} / \mathrm{serum}$ albumin ratio $=13,7$, reference range $<7,4$ ), but a significant reduction of cytokines levels. CSF RT-PCR for SARS-CoV-2 was still negative.

Neurologic syndrome was characterized by a status of akinetic mutism in keeping with the frontal slowing and hypometabolism shown at EEG and 18F-FDG-PET/CT, respectively. Structural lesions were excluded by brain MRI as well as autoimmune underlying causes by CSF and serum findings. Repeated negative CSF RT-PCR make SARSCov-2 neuroinvasion unlikely, although the presence of SARS-CoV-2 in CSF may be transient or untraceable.

Indeed, CSF analysis documented a downward trend of cytokines levels over time, from elevated IL-6 and IL-8 levels to normalization, which closely reflected the clinical improvement, suggesting a possible underlying cytokinemediated inflammatory process.

In particular, cytokine-mediated endothelial activation and increased brain-blood permeability are related to neurotoxicity $[5,6]$.

It is still not clearly understood in SARS-CoV-2 infection whether CSF neuroinflammation and subsequent neurotoxicity are induced by increased serum concentration of cytokines or directly driven by viral endothelial activation and blood-brain barrier disruption.

However, high CSF/serum IL-6 ratio detected in our patient at time of first lumbar puncture suggests that after a primary hit, cell-cytokine relationships may exponentially amplify resulting in intrathecal synthesis of neuroinflammatory mediators and glutamate hyper-excitatory neurotoxicity.

Predominant frontal symptoms with anterior slowing EEG findings and bilateral frontotemporal hypoperfusion have been similarly described in other cases of COVID19-related encephalopathy [1, 7]. In our case, ${ }^{18} \mathrm{~F}$-FDG-PET study confirmed the prevalent frontal involvement, showing spread frontal lobe hypometabolism.

Frontal syndrome may thus be considered the predominant feature of acute encephalopathy COVID-19-related. Moreover, cross-checked serum and CSF cytokines levels may represent a useful biomarker of early detection and progression of the neurologic involvement, as well as a ground for therapeutic recommendations and prognosis predictions.

Indeed, as the number of patients with COVID-19 increases worldwide, clinicians should be aware of predominant frontal lobe presentation and cytokine-mediated hyperinflammatory process. Further investigation is needed to evaluate efficacy of immunomodulatory therapies and interleukin/interleukin-receptor antagonist in COVID-19-related encephalopathy. 

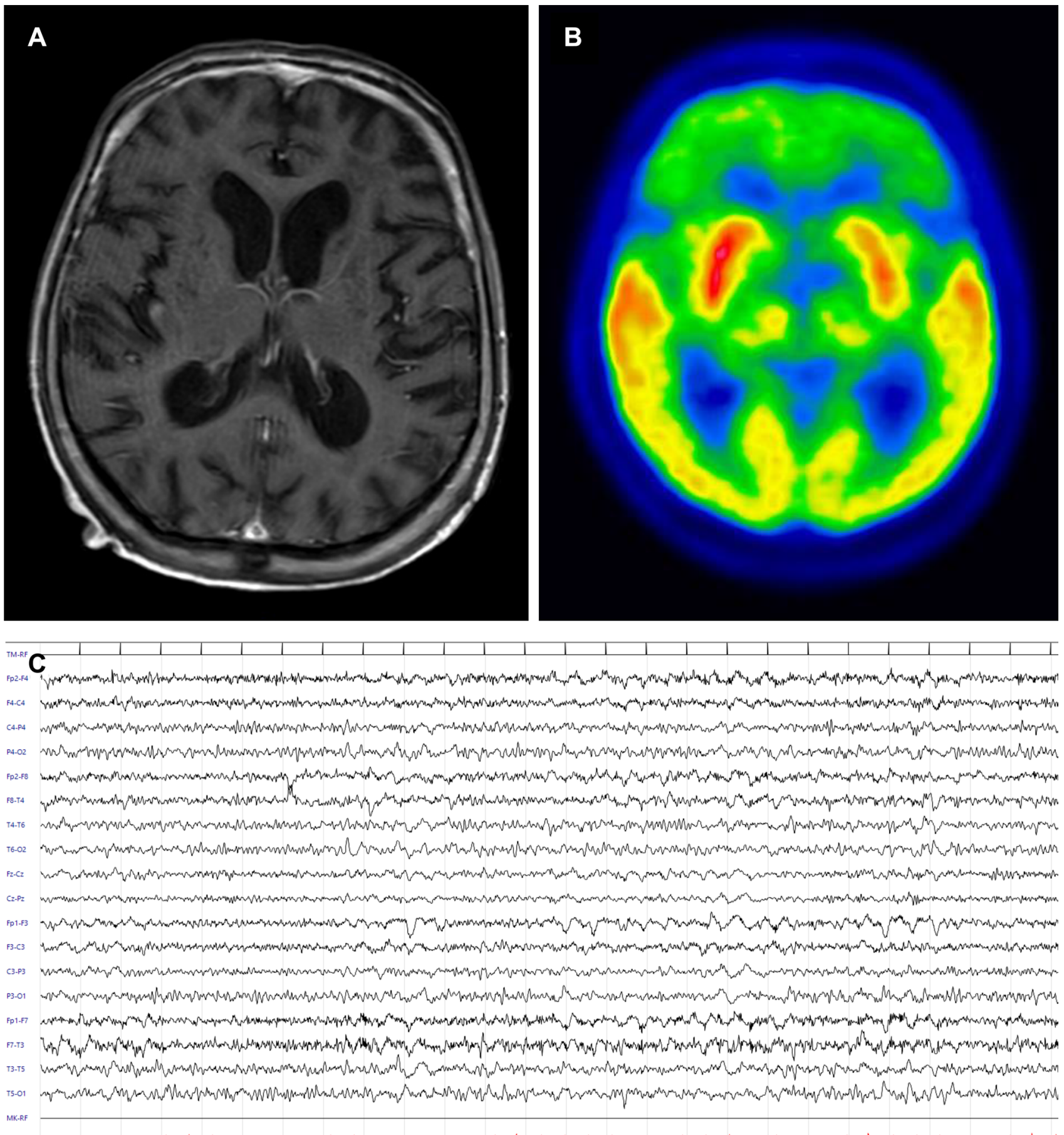

Fig. 2 Magnetic resonance imaging (MRI), ${ }^{18}$ F-FDG-PET/CT, and electroencephalography (EEG) findings. Normal MRI findings in MRI on T1 with gadolinium (a). ${ }^{18} \mathrm{~F}-\mathrm{FDG}$-PET/CT showing severe frontal lobe hypometabolism (b). EEG alterations observed at time of

Acknowledgements We are grateful to the patient for providing permission to publish this information. We thank Prof. M.C. Re, Dott.ssa first neurologic evaluation with prominent and bilateral frontal slowing (c). Electroencephalographic acquisition settings: 10-20 system, longitudinal montage. Recording speed: 30 s/page; sensitivity: $7 \mu \mathrm{V} /$ $\mathrm{mm}$; time constant: $0.1 \mathrm{~s}$; high-frequency filter: $70 \mathrm{~Hz}$

G. Rossini, Dott.ssa R. Mancini, Prof. P. Parchi, Dott. A. Paccagnella, and Dott.ssa S. Garrosi for their contributions in the performance and interpretation of laboratory analysis. 
Author contributions IC, VB, and RDA conceived and designed the study; IC, VB, and RDA analyzed the data and drafted a significant portion of the manuscript, figures, and table. LP, VA, LS, PM, LF, $\mathrm{RR}$, and SF acquired and analyzed data. IC, VB, RDA, PC, and MG contributed to revising the manuscript.

Funding The authors declare that they have nothing to report.

Data availability The authors take full responsibility for the data, the analysis, and interpretation of the research, and they have full access to all of the data.

\section{Compliance with ethical standards}

Conflicts of interest The authors declare that they have no conflict of interest.

Ethical standards This article does not contain any studies involving human participants performed by any of the authors.

Consent to participate Written informed consent was collected from the patient for the inclusion of deidentified clinical data in a scientific publication, in accordance with the Declaration of Helsinki.

Consent for publication All authors agreed with this final version.

\section{References}

1. Helms J, Kremer S, Merdji H et al (2020) Neurologic features in severe SARS-CoV-2 infection. N Engl J Med. https://doi. org/10.1056/nejmc2008597
2. Politi LS, Salsano E, Grimaldi M (2020) Magnetic resonance imaging alteration of the brain in a patient with coronavirus disease 2019 (COVID-19) and anosmia. JAMA Neurol. https://doi. org/10.1001/jamaneurol.2020.2125

3. Solomon IH, Normandin E, Bhattacharyya S et al (2020) Neuropathological features of Covid-19. N Engl J Med. https://doi. org/10.1056/NEJMc2019373(NEJMc2019373)

4. Mehta P, McAuley DF, Brown M et al (2020) COVID-19: consider cytokine storm syndromes and immunosuppression. Lancet 395:1033-1034. https://doi.org/10.1016/S0140-6736(20)30628-0

5. Santomasso BD, Park JH, Salloum D et al (2018) Clinical and biological correlates of neurotoxicity associated with car t-cell therapy in patients with B-cell acute lymphoblastic leukemia. Cancer Discov 8:958-971. https://doi.org/10.1158/2159-8290. CD-17-1319

6. Gust J, Hay KA, Hanafi LA et al (2017) Endothelial activation and blood-brain barrier disruption in neurotoxicity after adoptive immunotherapy with CD19 CAR-T cells. Cancer Discov 7:1404-1419. https://doi.org/10.1158/2159-8290.CD-17-0698

7. Pilotto A, Odolini S, Stefano Masciocchi S et al (2020) Steroidresponsive encephalitis in Covid-19 disease. Ann Neurol. https:// doi.org/10.1002/ana.25783 